\title{
Investigation of a kind of neutrino mass matrix
}

\author{
Chao-Shang Huang ${ }^{1 \dagger}$, Wen-Jun $\mathrm{Li}^{2 \ddagger}$ \\ 1.CAS Key Laboratory of Theoretical Physics, Institute of Theoretical Physics, \\ Chinese Academy of Sciences, Beijing 100190, China \\ 2.Physics school, Henan Normal University, Xinxiang 453007, China \\ E-mail: †csh@itp.ac.cn, ${ }^{\ddagger}$ liwj24@163.com
}

\begin{abstract}
We carry out diagonalization of a kind of Majorana neutrino mass matrix $M=M_{\nu} M_{\nu}^{\dagger}$ of which Real part and Imaginary part are commutative. For the kind of matrix M, it is shown in a modelindependent way that $\delta= \pm \pi / 2$ which implies the maximal strength of $\mathrm{CP}$ violation in neutrino oscillations and atmospherical mixing angle would be in the ranges, $\pi / 4<\theta_{23}<3 \pi / 4$, or $-3 \pi / 4<$ $\theta_{23}<-\pi / 4$. It is shown that the kind of Hermitian Majorana neutrino mass matrix $\mathrm{M}$ has only five real parameters and furthermore, only one free real parameter (A or D) if using the measured values of three mixing angles and mass squared differences as inputs.
\end{abstract}

PACS numbers:

\section{INTRODUCTION}

Massive data from solar, atmospheric, reactor and accelerator neutrino experiments facilitate research of neutrino mass and mixing in past thirty years. Knowledge of these magic particles has initially been grasped. However, the structure of neutrino mass matrix and their flavor mixing mechanism hidden behind are still not clear.

To understand the flavor structure of neutrinos is one of the outstanding problems in search for new physics. A series of attempts have been made to build the structure of neutrino mass matrix and their flavor mixing mechanism hidden behind. Some have considered inserting flavor symmetries to the standard model(SM), such as Tribimaximal mixing(TBM)[1],TM1 and TM2 mixings[2], texture zeros[3], vanishing cofactors [4], equalities [] and $\mu-\tau$ symmetry[7]. Recently, the case of neutrino mass matrix with the predicted atmospheric neutrino mixing angle $\theta_{23}$ near $45^{\circ}$ in type-I+II seesaw mechanism using non-Abelian flavor symmetry $A_{4}$ has been discussed [8]. The implementation of $U(1)_{L_{e}-L_{\tau}}$ gauge symmetry have been used to study the neutrino phenomenology within the framework of type-(I+II) seesaw [9]. Similar study with $S_{3} \otimes Z_{2}$ symmetry also has been finished[10]. In Ref. 11], a specific texture characterized by one equality between two independent elements in the neutrino mass matrix has been explored and it is noted that all the 15 possible one-Equality textures can accommodate the experimental data in the case of normal ordering, whereas only fourteen one-Equality patterns are viable in the inverted hierarchy type. Moreover, the authors in Ref.[12] have investigated the new texture of Majorana neutrino mass matrix under cosmic microwave background radiation and neutrinoless double beta decay measurements. Additionally, based on the cosmic microwave background temperature fluctuation and polarization measurements, Supernovae Ia luminosity distances, Baryon Acoustic Oscillation observations and determinations of the growth rate parameter, the most constraining bound have derived as $\sum m_{\nu}<0.09 \mathrm{eV}$ at $95 \% \mathrm{CL}[13]$. A model-independent way have been performed to constrain effective Majarana neutrino masses $\left|\left\langle M_{\alpha \beta}\right\rangle\right|(\alpha, \beta=e, \mu, \tau)$ with current experiment data, and got a few information of the absolute neutrino mass scale and one of the effective Majorana CP phases [14]. In Ref. 15], it is shown that two Majorana CP violating phases could be constrained from combination of neutrinoless double beta decay experimental constraint and cosmological constraint on effective Majorana neutrino mass. In addition, from the view of energy scales dependence, the neutrino mixing parameters under simple, ultraviolet-complete models have been discussed and several robust experimental signatures, some observable effects on neutrino experiments have been shown [16]. About the existing neutrino experiment factories, the authors in Ref.[17] have made an analysis of their systematic uncertainty and physics reach, and conceived a new generation of short-baseline cross section experiments.

Assuming that neutrino is Majorana fermion, one can express flavor eigen-states in terms of mass eigen-states:

$$
\left(\begin{array}{l}
\nu_{e} \\
\nu_{\mu} \\
\nu_{\tau}
\end{array}\right)=U_{P M N S}\left(\begin{array}{l}
\nu_{1} \\
\nu_{2} \\
\nu_{3}
\end{array}\right),
$$

where $U_{P M N S}$ is the neutrino mixing matrix [18]. The current experimental data of mixing angles, CP-violation phases 
and mass square differences for normal and inverted neutrino mass hierarchy have been obtained by means of neutrino oscillation experiments. ${ }^{1}$

In the flavoured basis, where charged lepton mass matrix is diagonal, Majorana neutrino matrix contains all the information regarding the leptonic $\mathrm{CP}$ violation at low energy. At present the measurements of the Dirac CP-violating phase $\delta$ show that $\delta_{C P} \approx \pi / 2$ or $\delta_{C P} \approx 3 \pi / 2$ most likely, which means that the leptonic CP violation is maximal most probably. What models of neutrino mass matrix can achieve the maximal CP violation? In this work we aim to search such models of neutrino mass matrix which can achieve the maximal $\mathrm{CP}$ violation and to investigate their features. The Ref. 21] gives us a clue to find such models, i.e., to realize $\Im\left(M_{12} M_{23} M_{31}\right)$, where $M=M_{\nu} M_{\nu}^{\dagger}$, reaches its maximum.

If the Real part and Imaginary part of a Hermitian matrix $M=M_{R}+i M_{I}$ are commutative:

$$
\left[M_{R}, M_{I}\right]=0
$$

it is easy to prove that

$$
M_{R}\left(\mathrm{i} M_{I}\right)^{\dagger}-\left(\mathrm{i} M_{I}\right) M_{R}^{\dagger}=0
$$

which leads to that $\Im\left(M_{12} M_{23} M_{31}\right)$ reaches its maximum. Therefore, we study the kind of Majorana neutrino mass matrix with the assumption (1.2). Of course, they are phenomenological models. One still needs to have more profound understanding of the flavor structure of neutrinos and flavor symmetry of leptons.

In the bases of the charged lepton mass matrix being diagonal and real, the Majorana neutrino mass matrix $M_{\nu}$ is a complex symmetric mass matrix which contains twelve real parameters. There are two ways to diagonalize the Majorana neutrino mass matrix. One way is directly to diagonalize $M_{\nu}[22]$, the other is to diagonalize the Hermitian matrix $M=M_{\nu} M_{\nu}^{\dagger}\left[23\right.$, 24]. In the case of diagonalization of $M_{\nu}, U_{P M N S}^{T} M_{\nu} U_{P M N S}=D_{\nu}$, as pointed out in reference 24], there is a parameter mismatching problem since $M_{\nu}$ contains twelve free real parameters but $U_{P M N S}$ and $D_{\nu}=\operatorname{diag}\left(m_{1}, m_{2}, m_{3}\right)$ totally consist of nine physical parameters. The problem can be solved by choosing a special flavor basis of three neutrino fields to factor out the three unphysical phases of $M_{\nu}[23$, 24]. In the case of diagonalization of $\mathrm{M}, U_{P M N S}^{+} M U_{P M N S}=D_{\nu}^{2}$, since $\mathrm{M}$ is a Hermitian matrix, it has advantages of less parameter numbers: 9 free real parameters. We use the special flavor basis of three neutrino fields which makes the three unphysical phases of $M_{\nu}$ be factored out. Using the $M^{\prime}=M_{\nu}^{\prime} M_{\nu}^{\prime+}$, where $M_{\nu}^{\prime}$ is the Majorana neutrino mass matrix in which the three unphysical phases of $M_{\nu}$ have been factored out and then $M_{\nu}^{\prime}$ has 9 free parameters, then $M^{\prime}$ has only 7 free real parameters since there are two constraint equations for the matrix elements of $M^{\prime}$. For the kind of Hermitian matrix $M^{\prime}$ that we study, there are 4 constraints so that $M^{\prime}$ has 5 free real parameters ${ }^{2}$. Therefore, $M_{\nu}^{\prime}$ has 5 free real parameters. In the paper we hereafter use $M_{\nu}$ and $\mathrm{M}$ in stead of $M_{\nu}^{\prime}$ and $M^{\prime}$ for simplicity. In the paper, we shall use the latter way, since a Hermitian matrix has advantages of less parameter numbers and two Majorana phases become irrelevant when investigating structure of $M_{\nu}$.

In the paper we diagonalize and investigate such kind of Hermitian neutrino mass matrix $M$ of which Real component and Imaginary part are commutative. The virtue of such matrix $M$ is, as pointed out above, with a few parameters, and the mass matrix $M_{\nu}$ is easily calculated, thus the study of such matrix $M$ should be helpful for investigating flavor mysteries and theoretical models in neutrino physics. In section 2 we show that a $3 \times 3$ Hermitian matrix only have five independent real parameters if its Real part and Imaginary part are commutative. The investigation of the kind of Hermitian neutrino mass matrix $M$ whose real and imaginary parts are commutative is carried out in sections 3 and two examples are presented in section 4 . Finally, in section 5 we give summary and conclusions. The detailed expressions of the solutions in section 4 are given in Appendix.

\footnotetext{
${ }^{1}$ For a review paper,see the Ref. [19] by Esteban et al. where the three flavor mixing angles, one CP-violating phase and two mass differences, which are extracted from a global analysis of current neutrino oscillation data, are listed in Table 3 . For more recent paper, see the Ref. [20].

2 The $\left[M_{R}, M_{I}\right]=0$ leads there are 4 constraints. It can be proved by a straightforward calculation that the two constraint equations for the matrix elements of $M^{\prime}$ above mentioned are same as the two of the four constraints.
} 


\section{INVESTIGATION OF A KIND OF HERMITIAN MATRIX}

Consider a $3 \times 3$ Hermitian matrix $H$ of which has the general pattern as

$$
H=\left(\begin{array}{ccc}
a_{1}+\mathrm{i} d_{1} & b_{1}+\mathrm{i} c_{1} & b_{2}+\mathrm{i} c_{2} \\
b_{4}+\mathrm{i} c_{4} & a_{2}+\mathrm{i} d_{2} & b_{3}+\mathrm{i} c_{3} \\
b_{5}+\mathrm{i} c_{5} & b_{6}+\mathrm{i} c_{6} & a_{3}+\mathrm{i} d_{3}
\end{array}\right)
$$

This Hermitian matrix $\mathrm{H}$ contains eighteen real parameters such that all $a_{i}, b_{j}, c_{j}, d_{i}(i=1,2,3, j=1, \ldots, 6)$ are real. It is well known that the Hermitian condition leads that $d_{i}=0, b_{i+3}=b_{i}$ and $c_{i+3}=-c_{i}(i=1,2,3)$. Thus, Eq.(2.1) now could expressed by two Real matrices of $H_{R}$ and $H_{I}$ :

$$
\begin{aligned}
H & =H_{R}+i H_{I} \\
H_{R} & =\left(\begin{array}{lll}
a_{1} & b_{1} & b_{2} \\
b_{1} & a_{2} & b_{3} \\
b_{2} & b_{3} & a_{3}
\end{array}\right), \\
H_{I} & =\left(\begin{array}{ccc}
0 & c_{1} & c_{2} \\
-c_{1} & 0 & c_{3} \\
-c_{2} & -c_{3} & 0
\end{array}\right),
\end{aligned}
$$

where $H_{R}$ is symmetric, $H_{I}$ is anti-symmetric. So the number of parameters is reduced from eighteen down to nine.

In this paper we study a kind of Hermitian matrix $H$ of which Real part and Imaginary part are commutative:

$$
\left[H_{R}, H_{I}\right]=0
$$

For such a kind of Hermitian matrix, it is straightforward to derive that their Real component and Imaginary component must satisfy a very interesting condition

$$
H_{R}\left(\mathrm{i} H_{I}\right)^{\dagger}-\left(\mathrm{i} H_{I}\right) H_{R}^{\dagger}=0 \text {. }
$$

Substituting Eq.(2.2) into Eq.(2.4), we will receive four equations:

$$
\begin{aligned}
b_{1} & =c_{2} c_{3}\left(a_{1}-a_{2}\right) / g, \\
b_{2} & =-c_{1} c_{3}\left(a_{1}-a_{2}\right) / g, \\
b_{3} & =c_{1} c_{2}\left(a_{1}-a_{2}\right) / g, \\
a_{3} & =a_{2}+\left(a_{1}-a_{2}\right) g_{1} / g,
\end{aligned}
$$

with $g=c_{2}^{2}-c_{3}^{2}, g_{1}=c_{2}^{2}-c_{1}^{2}$ which can be used to further reduce the parameter numbers from nine down to five. We come to the conclusion: a $3 \times 3$ Hermitian matrix of which Real component and Imaginary part are commutative has only five real parameters. It is worth noting that for a general Hermitian matrix the conclusion is not valid. As a matter of convenience we make the following choices: $a_{1}, a_{2}, c_{i}(i=1,2,3)$ are five real parameters, the other four real parameters can be determined from Eqs.(2.5-2.8).

\section{THE GENERAL NATURE OF NEUTRINO MASS MATRIX}

The symmetric Majorana neutrino mass matrix $M_{\nu}$ can be parameterized by

$$
M_{\nu}=\left(\begin{array}{lll}
a & b & c \\
b & d & e \\
c & e & f
\end{array}\right)
$$

which has nine real parameters. Although $M_{\nu}$ can be diagonalized by $U_{\text {PMNS }}$ [22], a simpler method is to diagonalize the Hermitian matrix $M=M_{\nu} M_{\nu}^{\dagger}$ [23],

$$
M=\left(\begin{array}{ccc}
A & B & C \\
B^{*} & D & E \\
C^{*} & E^{*} & F
\end{array}\right)
$$


with

$$
\begin{array}{ll}
A=|a|^{2}+|b|^{2}+|c|^{2}, & B=a b^{*}+b d^{*}+c e^{*} \\
C=a c^{*}+b e^{*}+c f^{*}, & D=|b|^{2}+|d|^{2}+|e|^{2} \\
E=b c^{*}+d e^{*}+e f^{*}, & F=|c|^{2}+|e|^{2}+|f|^{2} .
\end{array}
$$

As it is obvious, the Hermitian matrix $M$ has nine real parameters. In the paper we investigate a kind of Hermitian matrix $M=M_{\nu} M_{\nu}^{\dagger}$ of which Real component and Imaginary part are commutative so that we can use the results obtained in last section. We perform diagonalization by:

$$
U_{\mathrm{PMNS}}^{\dagger} M U_{\mathrm{PMNS}}=\left(\begin{array}{ccc}
m_{1}^{2} & 0 & 0 \\
0 & m_{2}^{2} & 0 \\
0 & 0 & m_{3}^{2}
\end{array}\right)
$$

where $m_{1}, m_{2}, m_{3}$ are real. From Eq.(3.2) and Eq.(3.4), one could get these relations:

$$
\begin{aligned}
m_{1}^{2} & =\xi_{1}-\chi t_{12}, \\
m_{2}^{2} & =\xi_{2}+\chi t_{12}=\xi_{1}+\frac{\chi}{t_{12}}, \\
m_{3}^{2} & =c_{13}^{2}\left(\xi_{3}+t_{13}^{2} A+t_{13}|y| 2 \cos \beta\right), \\
\xi_{1} & =A-|y| \cos \beta t_{13}, \\
\chi & =\frac{c_{23} \Re(B)-s_{23} \Re(C)}{c_{13}}, \\
\xi_{2} & =D+\Re(E)\left(-t_{23}\right), \\
\xi_{3} & =A+\frac{2|y|\left(\cos \left(2 \theta_{13}\right) \cos \beta+\mathrm{i} \sin \beta\right)}{\sin \left(2 \theta_{13}\right)},
\end{aligned}
$$

where

$$
\begin{aligned}
y & =s_{23} B+c_{23} C=|y| e^{\mathrm{i} \alpha}, \quad \beta=\alpha+\delta \\
\xi_{2}+\xi_{3} & =D+F
\end{aligned}
$$

and

$$
t_{i j}=\tan \theta_{i j}=\frac{\sin \theta_{i j}}{\cos \theta_{i j}}, s_{i j}=\sin \theta_{i j}, c_{i j}=\cos \theta_{i j},
$$

with $\theta_{i j}(\mathrm{i} \mathrm{j}=12,13,23)$ are mixing angles.

And one also has

$$
\begin{aligned}
\Im(B) & =t_{23} \Im(C), \\
\Im(E) & =s_{13} \chi \sin \delta, \\
\cos \delta & =\frac{\sin \left(2 \theta_{23}\right) \frac{F-D}{2}-\cos \left(2 \theta_{23}\right) \Re(E)}{s_{13} \chi} .
\end{aligned}
$$

It is important to note that Eq.(3.7) means that $\xi_{3}$ must be real. Then, from Eq.(3.11), we have the result of

$$
\sin \beta=0, \beta= \pm(n \pi), n=0,1,2 \ldots
$$

Furthermore, $\xi_{1}, \xi_{2}$ satisfies the constraint

$$
\left(\xi_{2}-\xi_{1}\right) \operatorname{tg}\left(2 \theta_{12}\right)=2 \chi
$$

According to the analysis of the previous section, a $3 \times 3$ Hermitian matrix of which Real component and Imaginary part are commutative has only five real parameters. That is, there are four Eqs.(2.5-2.8) which mean there are the four constraints as follows 


$$
\begin{aligned}
\Re(B) \Im(B) & =-\Re(C) \Im(C)=\Re(E) \Im(E), \\
A-D & =\frac{\Re(E) \Im(C)+\Re(C) \Im(E)}{\Im(B)}, \\
F-D & =\frac{\Re(C) \Im(B)+\Re(B) \Im(C)}{\Im(E)} .
\end{aligned}
$$

We can easily obtain the five independent real parameters by using the above four constraints (so $M_{\nu}$ has 8 real parameters) and transform them into two parameters plus three mixing angles. And we obtain two important conclusions when the four constraints are imposed.

From Eq.(3.14) and Eq.(3.19), it is straightforward to get the relation of

$$
\Re(C)=-t_{23} \Re(B) .
$$

Considering Eqs.(3.12) and (3.22), one can easily derive that the Real component of $y$ is zero:

$$
\Re(y)=0,
$$

i.e.,

$$
\cos \alpha=0, \alpha= \pm\left(\frac{\pi}{2}+m \pi\right), \quad m=0,1,2 \ldots
$$

provided that the modulus of $\mathrm{y}$ is not equal to zero, and

$$
\Im(y)=\frac{\Im(C)}{c_{23}} .
$$

Eqs.(3.12),(3.17) and Eq.(3.23) lead to

$$
\cos \delta=0, \delta= \pm\left(\frac{\pi}{2}+p \pi\right), \quad p=0,1,2 \ldots
$$

Therefore, due to Eq.(3.16), we have

$$
\frac{\sin \left(2 \theta_{23}\right) \frac{F-D}{2}-\cos \left(2 \theta_{23}\right) \Re(E)}{s_{13} \chi}=0 .
$$

Furthermore, The fourth equation, Eq.(3.21), of the constraints, can be simplified as

$$
F-D=\frac{\cos \left(2 \theta_{23}\right) \Im(C)}{c_{23} t_{13} \sin \delta} .
$$

Substituting Eq.(3.27) into Eq.(3.26), one has

$$
\cos \left(2 \theta_{23}\right)\left(\frac{\sin \left(2 \theta_{23}\right) \Im(C)}{2 c_{23} t_{13} \sin \delta}-\Re(E)\right)=0,
$$

since $s_{13} \chi$ is not equal to zero. To satisfy Eq.(3.28), there are the following cases:

$$
\text { a) } \cos \left(2 \theta_{23}\right)=0
$$

or

$$
\text { b) } \Re(E)=\frac{\sin \left(2 \theta_{23}\right) \Im(C)}{2 c_{23} t_{13} \sin \delta} \text {, }
$$

or both factors are zeros. 
We choose $A, \Im(C)$, and three mixing angles as five real parameters for specific. In terms of them, other real parameters could expressed as:

$$
\begin{aligned}
\Re(E) & =\frac{\Im(C) s_{23}}{t_{13} \sin \delta}, \\
\Re(B) & =\frac{\Im(E) c_{23}}{t_{13} \sin \delta}, \\
\Re(C) & =\frac{-\Im(E) s_{23}}{t_{13} \sin \delta}, \\
A-D & =\frac{-2|y| \cos \left(2 \theta_{13}\right) \cos \beta}{\sin \left(2 \theta_{13}\right)}+\frac{\Re(E)}{t_{23}} .
\end{aligned}
$$

Besides, combining Eqs.(3.19),(3.20) and Eq.(3.32), one could get the following relation by introducing the variable $g=\Im(C)^{2}-\Im(E)^{2}:$

$$
g=(A-D) \frac{\Im(C)\left(t_{13} \sin \delta\right)}{c_{23}} .
$$

Considering Eqs.(3.33) and (3.34), one obtains the equation:

$$
\Im(E)^{2}=|y|^{2}\left(2\left|c_{23}\right|^{2}-1+t_{13}^{2}\right) .
$$

Thus, the collection of Eqs.(3.14,3.27,3.30-33,3.35) explicitly gives the Hermitian Majorana neutrino mass matrix $M$ in terms of $A, \Im(C)$, and three mixing angles.

Moreover, we would like to determine the parameters $A$ and $\Im(C)$ by using mass squared differences. From Eqs.(3.53.7), we have

$$
\begin{aligned}
& \Delta m_{21}^{2}=\left|m_{2}^{2}-m_{1}^{2}\right|=\left|\frac{\chi\left(1+t_{12}^{2}\right)}{t_{12}}\right|, \\
& \Delta m_{31}^{2}=\left|m_{3}^{2}-m_{1}^{2}\right|=\left|\frac{|y| 2 \cos \beta}{\sin \left(2 \theta_{13}\right)}+\chi t_{12}\right| .
\end{aligned}
$$

Then we can use the measured values of mass squared differences to get the parameters $\Im(C)$ and $\chi$. However, Eqs.(3.8),(3.10),(3.18) lead to that $\chi$ depends $(A-D)$ so that one free parameter A or D still not fixed. Thus, there is only one parameter A (or D) in the Hermitian neutrino mass matrix M.

In summary of this section, we emphasize that $\delta= \pm \frac{\pi}{2}$ of Eq.(3.25) implies the maximal strength of CP violation in neutrino oscillations for given values of $\theta_{12}, \theta_{13}$ and $\theta_{23}$ (i.e., the leptonic Jarlskog parameter is maximal in this case [25]), which is a robust result for the kind of $\mathrm{M}$ that we study in the paper. The constraint, the first equation of (3.19), and the constraint, Eq.(3.14), due to diagonalization lead to $\cos \delta=0$. That is, the condition to have maximum $\mathrm{CP}$ violation is explicitly realized in this kind of models. One of possibilities to make Eq.(3.38) valid is $\cos \left(2 \theta_{23}\right)=0$. And in this case, Eq.(3.27) leads to the relation of $\mathrm{F}=\mathrm{D}$.

\section{MORE DISCUSSIONS ON MAJORANA NEUTRINO MASS MATRIX}

Eqs.(3.5-3.7) can be reduced to

$$
\begin{aligned}
& m_{1}^{2}=A-|y| \cos \beta t_{13}-\chi t_{12}, \\
& m_{2}^{2}=A-|y| \cos \beta t_{13}+\chi / t_{12} \\
& m_{3}^{2}=A+|y| \cos \beta / t_{13}
\end{aligned}
$$

After inputting experimental data of three mixing angles and two mass squared differences, all three masses, $m_{1}, m_{2}, m_{3}$, depend on the free parameter A (or D) which is only single free parameter in our case, as obviously seen from Eqs.(3.38-3.40). Because $M_{\nu}=U D_{\nu} U^{T}, D_{\nu}=\operatorname{diag}\left(m_{1}, m_{2}, m_{3}\right)$, $\mathrm{U}$ is the PMNS matrix, we have that the general Majorana neutrino mass matrix $M_{\nu}$ depends on the free parameter A (or D) only. That is, for the kind of Hermitian matrix $M=M_{\nu} M_{\nu}^{\dagger}$ of which Real component and Imaginary part are commutative, the general Majorana neutrino mass matrix $M_{\nu}$ is known except for a unknown parameter A after inputting the experimental data from 
neutrino oscillations. Such, one can not determine neutrino mass scale unless to input one more experimental data. For example, from experiments on the search for the neutrinoless double $\beta$ decay [26], one has $m_{\beta \beta} \leq 0.165 \mathrm{eV}$, which leads to $m_{1} \leq 0.15 \mathrm{eV}$ and then $A \leq 0.023 \mathrm{eV}^{2}$.

Based on the Eqs.(3.38-3.40), it is easy to get

1) $A>0$;

2)the constraints to model parameters from the neutrino mass hierarchy: for NMO (normal mass ordering), $\cos \beta=+1, \chi>0$; for IMO (inverted mass ordering), $\cos \beta=-1, \chi<0$.

Moreover, Eqs.(3.18), (3.33) lead to the sum rule of mixing angles

$$
\frac{2 \sqrt{2\left|c_{23}\right|^{2}-1+t_{13}^{2}}}{c_{13} t g\left(2 \theta_{12}\right)}+\epsilon=1,
$$

where $\epsilon= \pm 1$. Using data of the current global fits [19, 20], it follows that the sum rule is in agreement with data at 3 sigma level for both mass hierarchies. For the sake of satisfying Eq.(3.41), one has $\epsilon=+1$ so that $\cos \left(2 \theta_{23}\right)<0$, therefore,

$$
\pi / 4<\theta_{23}<3 \pi / 4,
$$

or

$$
-3 \pi / 4<\theta_{23}<-\pi / 4 .
$$

It is expected that the prediction, Eq.(3.42), will be verified in the future experiments.

\section{SOME EXAMPLES}

It is straightforward to obtain elements of the matrix $M_{\nu}$, which are useful to compare with theoretical models and give classification and some constraints on them, in terms of above five real parameters by solving Eq.(3.3). For the purpose of seeing what type of matrix $M_{\nu}$ belongs to the kind of matrix which is studied in this paper, we study some specific examples.

1)Model I

An extended version of $\mu-\tau$ symmetric mass matrix which accommodate non-zero $\theta_{13}$ along with maximal $\mathrm{CP}$ violation is given in $[22]$

$$
M_{\nu}=\left(\begin{array}{ccc}
a & b & -b^{*} \\
b & d & e \\
-b^{*} & e & d^{*}
\end{array}\right)
$$

here $a$ and $e$ are real. The neutrino mass matrix has been discussed in a number of works with discrete flavour symmetry models [7, 27 34]. If the matrix $M=M_{\nu} M_{\nu}^{\dagger}$ belongs the kind of matrix of which Real component and Imaginary part are commutative, we can get the expression of the matrix $M_{\nu}$ in terms of one free parameter and experimental data on neutrinos.

Applying Eqs.(3.3) and (4.1), one has the following equations:

$$
\begin{aligned}
\Re(B) & =-\Re(C), \\
\Im(B) & =\Im(C), \\
F & =D .
\end{aligned}
$$

Accordingly, using the method described in section 3, it follows that due to Eq.(3.23), one could receive $\cos \delta=0$ and due to Eq.(3.14), one has $t_{23}=1$ which means $\cos \left(2 \theta_{23}\right)=0$, as expected. Thus Eq.(3.3) can be reduced to the following six equations.

$$
\begin{aligned}
& -x_{1}^{2}+x_{2}^{2}+2 y_{1} z_{2}=a_{1}, \\
& 2 x_{1} x_{2}-2 y_{2} z_{2}=a_{2},
\end{aligned}
$$




$$
\begin{aligned}
& \left(z_{1}-z_{2}\right) x_{1}+x_{1} y_{1}+x_{2} y_{2}=b_{1}, \\
& \left(z_{1}-z_{2}\right) x_{2}+x_{1} y_{2}-x_{2} y_{1}=b_{2}, \\
& z_{1}^{2}+2\left(x_{1}^{2}+x_{2}^{2}\right)=c_{1}, \\
& x_{1}^{2}+x_{2}^{2}+y_{1}^{2}+y_{2}^{2}+z_{2}^{2}=c_{2},
\end{aligned}
$$

where $x_{1}=\Re(b), x_{2}=\Im(b), y_{1}=\Re(d), y_{2}=\Im(d), z_{1}=a, z_{2}=e$ and $a_{1}=\Re(E), a_{2}=\Im(E), b_{1}=\Re(B), b_{2}=$ $\Im(B), c_{1}=A, c_{2}=D$. From Eqs.(3.30),(3.35), we have the relation of $a_{1}$ and $a_{2}: a_{1}= \pm w^{2} a_{2}$ with $w=1 /\left(t_{13} \sqrt{2}\right)$. And Eqs.(3.31),(3.35) lead to the relation of $b_{1}$ and $b_{2}: b_{1}= \pm b_{2}$. So, the equations (4.4-4.9) can be simplified as:

$$
\left\{\begin{array}{c}
x_{1}^{2}-x_{2}^{2}=2 y_{1} z_{2} \mp w b_{2} \\
2 x_{1} x_{2}-2 y_{2} z_{2}=b_{2} /( \pm w) \\
\pm x_{2}-x_{1}= \pm y_{2} w+y_{1} /( \pm w) \\
\left( \pm b_{2}-b_{2}\right)=\left(z_{1}-z_{2}-y_{2}\right)\left(x_{1}-x_{2}\right)+y_{1}\left(x_{1}+x_{2}\right) \\
z_{1}^{2}+2\left(x_{1}^{2}+x_{2}^{2}\right)=c_{2}+b_{2}\left(w^{2}-1\right) /( \pm w) \\
x_{1}^{2}+x_{2}^{2}+y_{1}^{2}+y_{2}^{2}+z_{2}^{2}=c_{2}
\end{array}\right.
$$

Using the data of the neutrino mass squared differences, $\Delta m_{21}^{2}, \Delta m_{31}^{2}$, and mixing angles [19], we have the values of $a_{2}$ and $w: a_{2}=5.579 \times 10^{-5} \mathrm{eV}^{2}, w=4.676$. The Eqs.(4.4-4.9) are secondary simultaneous equations in six variables. We found two particular solutions of the aboved equations. Their detailed expressions are given in Appendix. To see the order of elements, which are called as the matrix parameters for simplicity hereafter, in the matrix $M_{\nu}$ (Eq.(4.1)), we list numerical value results of the two solutions below.

For the case of $y_{1}=y_{2}$, the matrix parameters are ${ }^{3}$

$$
\begin{aligned}
& a=-3.844 \times 10^{-3}, \quad b=(0.1716-3.751 \mathrm{i}) \times 10^{-2}, \\
& d=-8.023 \times 10^{-3}(1+\mathrm{i}), \quad e=1.150 \times 10^{-2} .
\end{aligned}
$$

So it is straightforward to get one solution of matrix $M_{\nu}$ from Eq.(4.1):

$$
M_{\nu}=\left(\begin{array}{lll}
-3.844 \times 10^{-3} & (0.1716-3.751 \mathrm{i}) \times 10^{-2} & (-0.1716-3.751 \mathrm{i}) \times 10^{-2} \\
(0.1716-3.751 \mathrm{i}) \times 10^{-2} & -8.023 \times 10^{-3}(1+\mathrm{i}) & 1.150 \times 10^{-2} \\
(-0.1716-3.751 \mathrm{i}) \times 10^{-2} & 1.150 \times 10^{-2} & -8.023 \times 10^{-3}(1-\mathrm{i})
\end{array}\right) .
$$

The another solution of mass matrix $M_{\nu}$ with $x_{1}=-x_{2}$ is:

$$
M_{\nu}=\left(\begin{array}{lll}
-5.324 \times 10^{-2} & 2.224 \times 10^{-4}(1-\mathrm{i}) & -2.224 \times 10^{-4}(1-\mathrm{i}) \\
2.224 \times 10^{-4}(1-\mathrm{i}) & (0.1173-5.376 \mathrm{i}) \times 10^{-2} & 5.198 \times 10^{-4} \\
-2.224 \times 10^{-4}(1-\mathrm{i}) & 5.198 \times 10^{-4} & 1.173 \times 10^{-3}+5.376 \times 10^{-2} \mathrm{i}
\end{array}\right) .
$$

Because there are indeed five variables in two particular solutions, no free parameter $\left(c_{1}\right.$ or $\left.c_{2}\right)$ remains in the solutions.

2)Model II

In this model, one neutrino mass matrix element vanishes [35]. So the mass matrix $M_{\nu}$ could be expressed as

$$
M_{\nu}=\left(\begin{array}{ccc}
0 & b & b^{*} \\
b & d & e \\
b^{*} & e & d^{*}
\end{array}\right)
$$

where $e$ is real.

Comparing with Eq.(3.1), we have $a=0, c=b^{*}, f=d^{*}$. Then the following relations are derived from Eq.(3.3)

$$
\begin{aligned}
& A=2|b|^{2}, \quad B=b d^{*}+b^{*} e, \quad C=b e+b^{*} d=B^{*}, \\
& D=|b|^{2}+|d|^{2}+e^{2}, \quad E=b^{2}+2 d e, \quad F=D .
\end{aligned}
$$

\footnotetext{
3 The unity of the matrix parameters is eV. All quantities in the following have the unity $\mathrm{eV}$ or $e V^{2}$.
} 
Beginning with $C=B^{*}$, one could derive that $\Re(B)=\Re(C), \Im(B)=-\Im(C)$ and considering Eq.(3.22), we have $\tan \theta_{23}=-1, \cos \left(2 \theta_{23}\right)=0$. Next, the relation $F=D, \cos \left(2 \theta_{23}\right)=0$, and Eq.(3.16) lead to the result of $\cos \delta=0$, as it is expected. Then, we have $\Re(E)=\sin \left(\theta_{23} \Im(C)\right) /\left(\tan \theta_{13} \sin \delta\right)$. Consequently, we could get the following equations:

$$
\begin{aligned}
& x_{1}\left(y_{1}+z_{2}\right)+x_{2} y_{2}=b_{1}, \\
& x_{2}\left(z_{2}-y_{1}\right)+x_{1} y_{2}=b_{2}, \\
& x_{1}^{2}-x_{2}^{2}+2 z_{2} y_{1}=a_{1}, \\
& -2\left(x_{1} x_{2}+z_{2} y_{2}\right)=a_{2}, \\
& y_{1}^{2}+y_{2}^{2}=c_{2}-c_{1} / 2,
\end{aligned}
$$

where $x_{1}$ and $x_{2}$ satisfy $x_{1}^{2}+x_{2}^{2}=c_{1} / 2$.

Two particular solutions of the mass matrix $M_{\nu}$ are derived and listed in Appendix. We give numerical results of the solutions in the following.

When $b_{1}=b_{2}$ is set up, one has the expression of $M_{\nu}$ :

$$
M_{\nu}=\left(\begin{array}{lll}
0 & (3.486-0.08753 \mathrm{i}) \times 10^{-2} & (3.486+0.08753 \mathrm{i}) \times 10^{-2} \\
(3.486-0.08753 \mathrm{i}) \times 10^{-2} & 7.309 \times 10^{-3}(1+\mathrm{i}) & 3.580 \times 10^{-4} \\
(3.486+0.08753 \mathrm{i}) \times 10^{-2} & 3.580 \times 10^{-4} & 7.309 \times 10^{-3}(1-\mathrm{i})
\end{array}\right) .
$$

When $b_{1}=-b_{2}$ holds, one has

$$
M_{\nu}=\left(\begin{array}{lll}
0 & (-3.493+0.07986 \mathrm{i}) \times 10^{-2} & (-3.493-0.07986 \mathrm{i}) \times 10^{-2} \\
(-3.493+0.07986 \mathrm{i}) \times 10^{-2} & -7.301 \times 10^{-3} \mathrm{i} & 7.301 \times 10^{-3} \\
(-3.493-0.07986 \mathrm{i}) \times 10^{-2} & 7.301 \times 10^{-3} & 7.301 \times 10^{-3} \mathrm{i}
\end{array}\right) \cdot(4 .
$$

Comparing with the result in Ref. [35], the order of numerical values of matrix elements is the same. Therefore, their model could be included into the kind of model that we investigated in this paper so that $\cos \delta=0$ must valid.

\section{SUMMARY AND CONCLUSIONS}

Using a very interesting equation inspired by an interesting condition [36], we have examined a kind of $3 \times 3$ Hermitian matrix of which Real component and Imaginary part are commutative and shown an important conclusion that the kind of $3 \times 3$ Hermitian matrix has only five real parameters. We have carried out diagonalization of the Hermitian Majorana neutrino mass matrix $M=M_{\nu} M_{\nu}^{\dagger}$ of which Real component and Imaginary part are commutative. Because the matrix elements of $\mathrm{M}$ have to satisfy four constraints we obtain in a model-independent way an important result, Dirac CP violation phase $\delta= \pm(\pi / 2+n \pi), n=0,1,2 \ldots$, a prediction, atmospherical mixing angle would be in the following ranges: $\pi / 4<\theta_{23}<3 \pi / 4$, or $-3 \pi / 4<\theta_{23}<-\pi / 4$, and some sum rule for mixing angles which is in agreement with data of the current global fits [19, 20] at 3 sigma level for both mass hierarchies. We have shown obviously that the mass matrix $M$ has only five real parameters and furthermore, only one free real parameter (A or D) if using the measured values of three mixing angles and mass squared differences as inputs. We have given matrix elements of neutrino mass matrix $M_{\nu}$ explicitly in some specific examples which should be helpful for investigating theoretical models in neutrino physics.

In the Ref.[37] authors examine the physics reach of the proposed medium baseline muon decay experiment MOMENT. To reach the precision of $\delta_{C P}$ at $10^{\circ}$ or better at $1 \sigma$ confidence level, they find it sufficient to combine the data of MOMENT, DUNE and T2HK. The authors in the Ref. [38] examine confidence intervals on $\delta_{C P}$ from the T2K plus Reactors fit in both the normal (NO) and inverted (IO) ordering. Their results (see Fig.4 in the reference) show that the values of $\delta_{C P}$ are in $(-2.42,-1.2)$ interval at the $68.27 \%$ confidence in the normal ordering and there are no values in the inverted ordering inside the $68.27 \%$ confidence interval.

It is expected that the precision measurement of $\delta_{C P}$ in the near future will help people to research the kind of neutrino models that we examined in the paper.

\section{Acknowledgement}

This research was supported in part by the Natural Science Foundation of China under grant numbers (No. 11847612 and No. 11875306). 
Appendix

This section gives the details of the Majorana neutrino mass matrix forms in Model I and Model II with parameters $a_{2}=5.579 \times 10^{-5} \mathrm{eV}^{2}, w=4.676$.

\section{The Model I}

In this model, we could get the Eqs.(4.9-4.14) from Eqs.(4.4-4.9) with $x_{1}=\Re(b), x_{2}=\Im(b), y_{1}=\Re(d), y_{2}=\Im(d)$, $z_{1}=a, z_{2}=e$ and $a_{1}=\Re(E), a_{2}=\Im(E), b_{1}=\Re(B), b_{2}=\Im(B), c_{1}=A, c_{2}=D$, and relations of $a_{1}= \pm w^{2} a_{2}, w=1 /\left(t_{13} \sqrt{2}\right), b_{2}=w a_{2}$.

Thus, two solutions could be obtained from Eqs.(4.9-4.14), one case is to satisfy the condition of $y_{1}=y_{2}$, and the other is that the condition of $x_{1}=-x_{2}$ holds.

$$
\begin{aligned}
\text { Case }(1): y_{1}=y_{2} \\
x_{1}=\sqrt{\frac{a_{2}\left(1+w^{2}\right)}{w^{4}-2 w^{2}-1}} \\
x_{2}=-w^{2} \sqrt{\frac{a_{2}\left(1+w^{2}\right)}{w^{4}-2 w^{2}-1}}, \\
y_{1}=-w \sqrt{\frac{a_{2}\left(1+w^{2}\right)}{w^{4}-2 w^{2}-1}}, \\
y_{2}=y_{1}, \\
z_{1}=\sqrt{\frac{a_{2}\left(1+w^{2}\right)}{w^{4}-2 w^{2}-1}} \cdot \\
\left.z_{2}=-\frac{w\left(1+w^{4}\right)}{\left(1+w^{2}\right)}+\frac{\left(1+w^{4}\right)\left(1+4 w^{2}+3 w^{4}-2 w^{6}\right)}{2 w\left(w^{4}-2 w^{2}-1\right)\left(1+w^{2}\right)}\right], \\
\sqrt{\frac{a_{2}\left(1+w^{2}\right)}{w^{4}-2 w^{2}-1}}
\end{aligned}
$$

With $a_{2}=5.579 \times 10^{-5}, w=4.676$, the numerical results could be listed:

$$
\begin{aligned}
& x_{1}=1.716 \times 10^{-3}, x_{2}=-3.751 \times 10^{-2}, \\
& y_{1}=y_{2}=-8.023 \times 10^{-3}, \\
& z_{1}=-3.844 \times 10^{-3}, \quad z_{2}=1.15 \times 10^{-2} .
\end{aligned}
$$

According to the relations of $x_{1}=\Re(b), x_{2}=\Im(b), y_{1}=\Re(d), y_{2}=\Im(d), z_{1}=a, z_{2}=e$, one could calculate the matrix parameters of $a, b, d, e$ :

$$
\begin{aligned}
& a=-3.844 \times 10^{-3}, \quad b=1.716 \times 10^{-3}-3.751 \times 10^{-2} \mathrm{i} \\
& d=-8.023 \times 10^{-3}(1+\mathrm{i}), \quad e=1.15 \times 10^{-2} .
\end{aligned}
$$

The form of mass matrix $M_{\nu}$ could be written as (4.15) in section 4 .

$$
\text { Case }(2): x_{1}=-x_{2}
$$

Similarly, we could get the following expressions:

$$
\begin{aligned}
& x_{1}=-x_{2}, \\
& x_{2}=-\frac{b_{2}}{a_{1}^{2}+4 b_{2}^{2}} \cdot M, \\
& y_{1}=-\frac{b_{2}}{x_{2}} \\
& y_{2}=\left(\left(a_{1}^{2}+4 b_{2}^{2}\right) \cdot M\right)^{-1} .
\end{aligned}
$$




$$
\begin{aligned}
& {\left[4 b_{2}^{2} \sqrt{\left(2 a_{2}+c_{1}\right)\left(a_{1}^{2} c_{1}-8 a_{2} b_{2}^{2}\right)}-a_{1}^{3} a_{2}-4 a_{1} b_{2}^{2}\right],(A .12) } \\
z_{1}= & {\left[a_{1}^{3} c_{1}+4 b_{2}^{2} \sqrt{\left(2 a_{2}+c_{1}\right)\left(a_{1}^{2} c_{1}-8 a_{2} b_{2}^{2}\right)}\right.} \\
& \left.-a_{1}\left(16 a_{2} b_{2}^{2}+4 b_{2}^{2} c_{1}+a_{1} \sqrt{\left(2 a_{2}+c_{1}\right)\left(a_{1}^{2} c_{1}-8 a_{2} b_{2}^{2}\right)}\right)\right] . \\
& \left(\left(a_{1}^{2}+4 b_{2}^{2}\right) \cdot M\right)^{-1}, \\
z_{2}= & \frac{a_{1} \cdot M}{\left.2\left(a_{1}^{2}+4 b_{2}^{2}\right)\right)}, \\
M= & {\left[2 a_{1}^{2}\left(a_{2}+c_{1}\right)\right.} \\
& \left.-2\left(4 a_{2} b_{2}^{2}+a_{1} \sqrt{\left(2 a_{2}+c_{1}\right)\left(a_{1}^{2} c_{1}-8 a_{2} b_{2}^{2}\right)}\right)\right]^{-\frac{1}{2}}, \\
c_{1}= & \left(1+12 w^{2}+42 w^{4}+72 w^{6}+81 w^{8}+28 w^{10}+8 w^{12}\right) \\
& \left(a_{2}+2 b_{2} w+b_{2} w^{3}\right) /\left[4 w^{2}\left(1+w^{2}\right)^{3}\left(w^{4}-2 w^{2}-1\right)\right] \\
- & \frac{a_{2}+6 b_{2} w+14 b_{2} w^{3}+16 b_{2} w^{5}+5 b_{2} w^{7}+2 b_{2} w^{9}}{\left(1+w^{2}\right)\left(-1-2 w^{2}+w^{4}\right)} .
\end{aligned}
$$

With inputs of $w$ and $a_{2}$, we get $c_{1}=2.835 \times 10^{-3}$ and the numerical results are given

$$
\begin{aligned}
& x_{1}=2.224 \times 10^{-4}, \quad x_{2}=-2.224 \times 10^{-4}, \\
& y_{1}=1.173 \times 10^{-3}, \quad y_{2}=-5.376 \times 10^{-2}, \\
& z_{1}=-5.324 \times 10^{-2}, \quad z_{2}=5.198 \times 10^{-4} .
\end{aligned}
$$

The matrix parameters of $a, b, d, e$ are

$$
\begin{aligned}
& a=-5.324 \times 10^{-2}, \quad b=2.224 \times 10^{-4}(1-\mathrm{i}), \\
& d=10^{-2} \times(0.1173-5.376 \mathrm{i}), \quad e=5.198 \times 10^{-4} .
\end{aligned}
$$

The mass matrix $M_{\nu}$ could see Eq.(4.16) in section 4 .

\section{The Model II}

In this model, one neutrino mass matrix element vanishes. According to the analysis in section 4, one could solve Eqs.(4.18-4.22) and get two solutions with

$$
\begin{aligned}
a_{1} & =\Re(E), a_{2}=\Im(E), b_{1}=\Re(B), b_{2}=\Im(B), \\
c_{1} & =A, c_{2}=D, x_{1}=\Re(b), x_{2}=\Im(b), \\
y_{1} & =\Re(d), y_{2}=\Im(d), z_{2}=e, z_{1}=a=0, \\
w & =1 /\left(t_{13} \sqrt{2}\right), a_{1}=w b_{2}, b_{2}=w a_{2} .
\end{aligned}
$$

Here two cases are discussed with $b_{1}= \pm b_{2}$ established.

$$
\begin{aligned}
& \text { Case }(1): b_{1}=b_{2} \\
& x_{1}=u-\sqrt{\frac{u^{2}-a_{1}+a_{2}}{2}}, \\
& x_{2}=-\sqrt{\frac{u^{2}-a_{1}+a_{2}}{2}}, \\
& y_{1}=\frac{b_{2} u}{a_{1}+a_{2}},
\end{aligned}
$$




$$
\begin{aligned}
y_{2}= & y_{1}, \\
z_{2}= & \frac{2 b_{2} \sqrt{\frac{u^{2}-a_{1}+a_{2}}{2}}}{a_{1}+a_{2}} \\
u= & \sqrt{\frac{M_{1}+\sqrt{M_{2}}}{M_{3}}}, \\
M_{1}= & a_{1}^{4} a_{2}+a_{2}^{5}-4 a_{2}^{3} b_{2}^{2}+4 a_{2} b_{2}^{4}+4 a_{1}^{3}\left(a_{2}^{2}-b_{2}^{2}\right) \\
& +6 a_{1}^{2}\left(a_{2}^{3}-2 a_{2} b_{2}^{2}\right)+4 a_{1}\left(a_{2}^{4}-3 a_{2}^{2} b_{2}^{2}+b_{2}^{4}\right), \\
M_{2}= & \left(a_{1}+a_{2}\right)^{2}\left(a_{1}^{2}+2 a_{1} a_{2}+a_{2}^{2}-2 b_{2}^{2}\right)^{2} \\
& \cdot\left(a_{1}^{4}+2 a_{1}^{3} a_{2}+2 a_{1}^{2}\left(a_{2}^{2}-2 b_{2}^{2}\right)+\left(a_{2}^{2}-2 b_{2}^{2}\right)^{2}\right. \\
& \left.+2 a_{1}\left(a_{2}^{3}-4 a_{2} b_{2}^{2}\right)\right), \\
M_{3}= & a_{1}^{4}+4 a_{1}^{3} a_{2}+a_{2}^{4}-8 a_{2}^{2} b_{2}^{2}+8 b_{2}^{4} \\
& +a_{1}^{2}\left(6 a_{2}^{2}-8 b_{2}^{2}\right)+4 a_{1}\left(a_{2}^{3}-4 a_{2} b_{2}^{2}\right) .
\end{aligned}
$$

Inputting the values of $w$ and $a_{2}$, we have $u=3.574 \times 10^{-2}$ and then the numerical solution is:

$$
\begin{aligned}
& x_{2}=-8.753 \times 10^{-4}, x_{1}=3.486 \times 10^{-2}, \\
& y_{2}=y_{1}=7.309 \times 10^{-3}, \\
& z_{2}=3.580 \times 10^{-4} .
\end{aligned}
$$

Consequently the results of mass matrix parameters are

$$
\begin{aligned}
b & =3.486 \times 10^{-2}-8.753 \times 10^{-4} \mathrm{i}, \\
d & =7.309 \times 10^{-3}(1+\mathrm{i}), \\
e & =3.580 \times 10^{-4} .
\end{aligned}
$$

The mass matrix could be written as Eq.(4.23) in section 4 .

$$
\text { Case (2): } b_{1}=-b_{2}
$$

In the the same way, the following solution could be gotten:

$$
\begin{aligned}
& x_{2}=\sqrt{\frac{-a_{1}+\sqrt{a_{1}^{2}+a_{2}^{2}}}{2}}, \\
& x_{1}=\sqrt{\frac{-a_{1}+\sqrt{a_{1}^{2}+a_{2}^{2}}}{2}}+u, \\
& y_{1}=0, \\
& y_{2}=\frac{b_{2}}{u} \\
& z_{2}=-y_{2}, \\
& u=\frac{a_{1}-a_{2}-\sqrt{a_{1}^{2}+a_{2}^{2}}}{\sqrt{2\left(\sqrt{a_{1}^{2}+a_{2}^{2}}-a_{1}\right)}} .
\end{aligned}
$$

With the inputs of $w=4.676, a_{2}=5.579 \times 10^{-5}$, one has $b_{2}=2.609 \times 10^{-4}, a_{1}=1.22 \times 10^{-3}, u=-3.573 \times 10^{-2}$ and the numerical results of the solution are given:

$$
\begin{aligned}
& x_{1}=-3.493 \times 10^{-2}, \quad x_{2}=7.986 \times 10^{-4}, \\
& y_{1}=0, y_{2}=-7.301 \times 10^{-3}, \\
& z_{2}=7.301 \times 10^{-3} .
\end{aligned}
$$

Thus, the numerical values of mass matrix parameters are presented

$$
\begin{aligned}
b & =-3.493 \times 10^{-2}+7.986 \times 10^{-4} \mathrm{i}, \\
d & =-7.301 \times 10^{-3} \mathrm{i}, \\
e & =7.301 \times 10^{-3} .
\end{aligned}
$$

The mass matrix $M_{\nu}$ could be written as Eq.(4.24) in section 4 . 


\section{References}

[1] P. F. Harrison, D. H. Perkins and W. G. Scott, Phys. Lett. B 530, (2002) 167, arXiv:0202074[hep-ph]; P. F. Harrison and W. G. Scott, Phys. Lett. B 535, (2002) 163-169, arXiv:0203209[hep-ph]; Z. z. Xing, Phys. Lett. B 533, (2002) 85-93, arXiv:0204049 [hep-ph]; J. Ganguly, R. S. Hunid, arXiv:2107.07275 hep-ph].

[2] X. G. He and A. Zee, Phys. Lett. B 645, (2007) 427, arXiv:0607163[hep-ph]; X. G. He and A. Zee, Phys. Rev. D 84, (2011) 0530, arXiv:1106.4359 hep-ph]; Carl H. Albright, Werner Rodejohann, Eur. Phys. J. C 62, (2009) 599-608, arXiv:0812.0436 hep-ph]; Carl H. Albright, Alexander Dueck, Werner Rodejohann, Eur. Phys. J. C 70, (2010) 1099-1110, arXiv:1004.2798 hep-ph].

[3] Paul H. Frampton, Sheldon L. Glashow and Danny Marfatia, Phys. Lett. B 536, 79 (2002), arXiv:0201008[hep-ph]; Zhizhong Xing, Phys. Lett. B 530, (2002) 159, arXiv:0201151[hep-ph]; Bipin R. Desai, D. P. Roy and Alexander R. Vaucher, Mod. Phys. Lett. A 18, (2003) 1355, arXiv:0209035[hep-ph]; A. Merle, M. Singh, G. Ahuja and M. Gupta, PTEP 2016, no.12, (2016) 123B08, arXiv:1603.08083 hep-ph], D. Borah, M. Ghosh, S. Gupta and S. K. Raut, Phys. Rev. D 96, (2017) 055017, arXiv:1706.02017 hep-ph].

[4] L. Lavoura, Phys. Lett. B 609, (2005) 317, arXiv:0411232[hep-ph]; E. I. Lashin and N. Chamoun, Phys. Rev. D 78, (2008) 073002, arXiv:0708.2423 hep-ph]; E. I. Lashin, N. Chamoun, Phys. Rev. D 80, (2009) 093004, arXiv:0909.2669 hep-ph]; W. Wang, Phys. Lett. B 733, (2014) 320, Erratum:[Phys. Lett. B 738, (2014) 524], arXiv:1401.3949 hep-ph]; W. Wang, Phys. Rev. D 90, no. 3, (2014) 033014, arXiv:1402.6808 hep-ph].

[5] S. Dev, R. R. Gautam and L. Singh, Phys. Rev. D 87, (2013) 073011, arXiv:1303.3092 hep-ph].

[6] S. Kaneko, H. Sawanaka and M. Tanimoto, JHEP 0508, (2005) 073, arXiv:0504074[hep-ph]; S. Dev, S. Verma and S. Gupta, Phys. Lett. B 687, (2010) 53-56, arXiv:0909.3182 hep-ph]; J. Y. Liu and S. Zhou, Phys. Rev. D 87, no.9, (2013) 093010, arXiv:1304.2334 hep-ph]; W. Wang, Eur. Phys. J. C 73, (2013) 2551, arXiv:1306.3556 hep-ph]; S. Dev, R. R. Gautam and L. Singh, Phys. Rev. D 88, (2013) 033008, arXiv:1306.4281 hep-ph]; S. Dev and D. Raj, Nucl. Phys. B 957, (2020) 115081, arXiv:2006.12019 hep-ph].

[7] S. F. King and C. C. Nishi, Phys. Lett. B 785, (2018) 391, arXiv:1807.00023 hep-ph]; C. C. Nishi, et al., JHEP B 09, (2018) 042, arXiv:1806.07412 hep-ph].

[8] R. R. Gautam, Sanjeev Kumar, Phys. Lett. B 820, (2021) 136504, arXiv:2107.01526 hep-ph].

[9] Mitesh Kumar Behera, et al., arXiv:2108.04066 [hep-ph].

[10] J. D. García, J. C. Gómez-Izquierdo, arXiv:2108.00317 hep-ph].

[11] A. Ismael, et al., Nucl. Phys. B 971, (2021) 115541, arXiv:2102.08326 hep-ph].

[12] Yuta Hyodo, and Teruyuki Kitabayashi, Progress of Theoretical and Experimental Physics, B 123, (2021) 08, arXiv:2105.08210 hep-ph].

[13] Eleonora Di Valentino, et al., Phys. Rev.D 104, (2021) 083504, arXiv:2106.15267[hep-ph].

[14] Ce-ran Hu, ZHi-Zhong Xing, Nucl. Phys. B 971, (2021) 115521, arXiv:2108.00986 hep-ph]; H. Georgi and S. L. Glashow, Phys. Rev. D 61, (2000) 097301, hep-ph/9808293 G. C. Branco et al, Phys. Rev. Lett. 82 (1999) 683, hep-ph/9810328 R. Adhikari and G. Rajasekaran, Phys. Rev. D 61, (2000) 031301, hep-ph/9812361

[15] Zhi-zhong Xing, Ye-Ling Zhou, Phys. Rev D 88, (2013) 033002, arXiv:1305.5718; Zhi-zhong Xing, Physics Reports 854 (2020) 1, 1909.09610 [hep-ph].

[16] K. S. Babu, et al., arXiv:2108.11961 hep-ph].

[17] A. Branca, et al., Symmetry 13, 9, (2021) 1625, arXiv:2108.12212 hep-ph].

[18] Particle Data Group, K. Nakamura et al., J. Phys. G 37, (2010) 075021; PMNS matrix was been introduced in B. Pontecorvo, Zh. Eksp. Teor. Fiz., 34 (1958) 247, (Sov. Phys. JETP, 7, (1958) 172); Zh. Eksp. Teor. Fiz. 53, (1967) 1717, (Sov. Phys. JETP, 26, (1968) 984); Z. Maki, M. Nakagawa, S. Sakata, Prog. Theor. Phys. 28, (1962) 870.

[19] Ivan Esteban, et al., JHEP, 09 (2020) 178, arXiv:2007.14792 hep-ph].

[20] NuFIT, http://www.nu-fit.org/?q=node/8; NuFIT 5.1 (2021), www.nu-fit.org.

[21] M. Singh, Prog Theor.Exp.Phys., B04 (2020) 093, arXiv:2006.09687[hep-ph].

[22] Chandan Duarah, arXiv:1905.07910 hep-ph].

[23] Ichiro Aizawa, Masaki Yasuè, Phys. Lett. B 607, (2005) 267, arXiv:0409331[hep-ph];

[24] Xing Zhizhong, Ye-Ling Zhou, Phys. Lett. B 693, (2010) 584, arXiv:1008.4906 hep-ph].

[25] C. Jarlskog, Phys. Rev. Lett. 55, (1985) 1039; Z.Z. Xing, Phys. Lett. B 679, (2009) 111; and references therein.

[26] A. Gando et al. (KamLAND-Zen), Phys. Rev. Lett. 117, 082503 (2016), [Addendum: Phys.Rev.Lett. 117, 109903 (2016)], arXiv:1605.02889 [hep-ex].

[27] P. F. Harrison, W. G. Scott, Phys. Lett. B 547, (2002) 219, arXiv:0210197[hep-ph].

[28] K. S. Babu, E. Ma and J. W. F. Valle, Phys. Lett. B 552, (2003) 207, arXiv:0206292[hep-ph].

[29] E. Ma, Phys. Lett. B 583, (2004) 157, arXiv:0308282[hep-ph].

[30] W. Grimus, L. Lavoura, Phys. Lett. B 579, (2004) 113, arXiv:0305309[hep-ph].

[31] X.-G. He, Chin. J. Phys. 53, (2015) 100101, arXiv:1504.01560v3[hep-ph].

[32] T. Fukuyama, PTEP 2017, (2017) 3, 033B11, arXiv:1701.04985 1 [hep-ph].

[33] Z.-Z. Xing, Z.-H. Zhao, Rep. Prog. Phys. 79, (2016) 7:076201, arXiv:1512.04207[hep-ph]. 
[34] E. Ma, A. Natale, O. Popov, Phys. Lett. B 746, (2015) 114, arXiv:1502.08023 hep-ph]. JHEP 09 (2018) 042, arXiv:1806.07412,

[35] Zhi-Cheng Liu, Chong-Xing Yue and Zhen-hua Zhao, Phys. Rev. D 99, (2019) 075034, arXiv:1808.06837[hep-ph].

[36] G. C. Branco, A. J. Buras, and J. M. Gerard, Nucl. Phys. B 259, (1985) 306.

[37] Jian Tang, Sampsa Vihonen, JHEP, 2019, (2019) 12:130, arXiv:1909.01548 hep-ph].

[38] The T2K Collaboration, Nature 580, (2020) 339, arXiv:1910.03887[hep-ex]. 